\title{
Posterior-only surgical correction of dystrophic scoliosis in 31 patients with neurofibromatosis Type 1 using the multiple anchor point method
}

\author{
Ang Deng, MD, Hong-Qi Zhang, MD, Ming-Xing Tang, MD, Shao-Hua Liu, MD, Yu-Xiang Wang, MD, \\ and Qi-Le Gao, MD
}

Department of Spine Surgery, Xiangya Hospital of Central South University, ChangSha, China

OBJECTIVE The objective of this study was to evaluate the clinical efficacy of posterior-only surgical correction of dystrophic scoliosis in patients with neurofibromatosis Type 1 (NF1) using a multiple anchor point method (MAPM).

METHODS From 2005 to 2014, 31 patients (mean age 13.5 years old, range 10-22 years old) suffering from dystrophic scoliosis associated with NF1 underwent posterior-only surgical correction using a MAPM. The apex of the deformity was thoracic $(n=25)$, thoracolumbar $(n=4)$, and lumbar $(n=2)$. The mean preoperative coronal Cobb angle was $69.1^{\circ}$ (range $48.9^{\circ}-91.4^{\circ}$ ). The mean Cobb angle on the side-bending radiograph of the convex side was $58.2^{\circ}$ (range $40^{\circ}-$ $79.8^{\circ}$ ). The mean flexibility and apical vertebral rotation (AVR) were $15.6 \%$ (range $8.3 \%-28.2 \%$ ) and $2.5^{\circ}$ (range $2^{\circ}-3^{\circ}$ ), respectively. The mean angle of sagittal kyphosis was $58.3^{\circ}$ (range $34.1^{\circ}-79.6^{\circ}$ ).

RESULTS The mean follow-up period was 53 months (range 12-96 months). The mean postoperative coronal Cobb angle was $27.4^{\circ}$ (range $16.3^{\circ}-46.7^{\circ}$ ). Postoperatively, the mean AVR and angle of sagittal kyphosis were $1.2^{\circ}$ (range $1^{\circ}-2^{\circ}$ ) and $22.4^{\circ}$ (range $4.2^{\circ}-36.3^{\circ}$ ), respectively. All patients showed good correction of all indices postoperatively. The mean postoperative correction rate was $58.7 \%$ (range $46.3 \%-74.1 \%$ ). At the final follow-up evaluation, the corrective loss rate of the Cobb angle was only 2.3\%. Only 1 patient required revision surgery. No severe complications such as spinal cord, neural, or large vascular injury occurred during the operation.

CONCLUSIONS Posterior-only surgical correction of dystrophic scoliosis in patients with NF1 using a MAPM could yield satisfactory clinical efficacy of correction and fusion.

http://thejns.org/doi/abs/10.3171/2016.7.PEDS16125

KEY WORDS neurofibromatosis; dystrophic scoliosis; posterior-only surgical correction; multiple anchor point method; spine

\begin{abstract}
O CoLIOSIS is considered to be the most common skeletal manifestation in patients with neurofibromatosis Type 1 (NF1). This scoliosis can be divided into 2 categories: nondystrophic and dystrophic. Nondystrophic scoliosis is similar to idiopathic scoliosis, and should be treated as such. However, dystrophic scoliosis is a characteristic curve pattern described as a short, sharp, angular curve, usually accompanied by kyphosis and associated with obvious dystrophic changes. Furthermore, it is very difficult to place posterior pedicle screws and surgically correct spinal deformities due to severe dystrophic vertebral deformity, very thin pedicles, and vertebral rotation. Therefore, the treatment strategies are more complicated
\end{abstract}

and significantly different from those of other types of scoliosis. ${ }^{59}$ However, there are no clinical trials analyzing, comparing, or contrasting surgical approaches for the treatment of dystrophic scoliosis associated with NF1. A high incidence of complications, including internal fixation dislodgement, breakage, pseudarthrosis, and misplaced pedicle screws, has been observed. Anteroposterior instrumented fusion has been used in most patients previously, but treatment strategies remain controversial. The aim of this work was to retrospectively evaluate the clinical efficacy of posterior-only surgical correction using the multiple anchor point method (MAPM) for dystrophic scoliosis in patients with NF1. 


\section{Methods}

\section{Study Population}

From 2005 to 2014, 31 patients (24 boys and 7 girls, mean age 13.5 years, range 10-22 years) who suffered from dystrophic scoliosis associated with NF1, were treated at the Department of Spine Surgery, Xiangya Hospital of Central South University. All the patients had received a diagnosis of NF because of the presence of at least 2 of the clinical criteria defined by the Consensus Development Conference of the NIH on NF1 (1987). ${ }^{11}$ Before being referred to our department, 7 patients underwent unsuccessful bracing therapy for an average period of 8 months (range 6 months-1 year). In all patients, radiography, CT, and MRI examinations revealed typical dystrophic changes, including vertebral scalloping $(n=27)$, rib penciling $(n=23)$, vertebral wedging $(n=28)$, short angular curve $(n=31)$, elongated and thin pedicles $(n=31)$, intervertebral foraminal enlargement $(n=15)$, and dural ectasia $(n=26)$. The apex of the deformity was thoracic ( $n$ $=25)$, thoracolumbar $(n=4)$, and lumbar $(n=2)$. The mean preoperative coronal Cobb angle was $69.1^{\circ}$ (range $48.9^{\circ}-$ $91.4^{\circ}$ ). The mean Cobb angle on the side-bending radiograph on the convex side was $58.2^{\circ}$ (range $40^{\circ}-79.8^{\circ}$ ). The mean flexibility and apical vertebral rotation were $15.6 \%$ (range $8.3 \%-28.2 \%$ ) and $2.5^{\circ}$ (range $2^{\circ}-3^{\circ}$ ), respectively. The mean angle of sagittal kyphosis was $58.3^{\circ}$ (range $34.1^{\circ}-79 \cdot 6^{\circ}$ ). Clinical examination included a thorough neurological examination. No obvious neural dysfunction was found in any patient.

The indications for posterior-only surgical correction using a MAPM were based on: 1) coronal Cobb angle greater than $20^{\circ}$; 2) consideration of the magnitude and progression of the spinal deformity; 3 ) obvious dystrophic changes; and 4) age greater then 10 years old. Exclusion criteria were based on: 1) age less than 10 years old; 2) intraspinal tumor; and 3) incomplete clinical and/or radiological documentation at follow-up. The Ethics Committee at Xiangya Hospital of Central South University approved the study.

\section{Operative Procedure}

During the operation, somatosensory evoked potentials and the wake-up test were used to monitor the spinal cord function, thereby preventing neural complications. Through a midline incision, the posterior spinal elements including lamina, facet joints, transverse processes, and costotransverse articulations were exposed. Then, posterior-only surgical correction using a MAPM was performed. Soft-tissue contractures and facet joint capsules on the concave side were completely released. Intertransverse ligaments and costotransverse articulation ligaments at the rigid segments were also excised. If necessary, parts of the ribs and transverse processes at the apical vertebrae were removed. Rod rotation, compression, distraction, and derotation were used for correction of scoliosis after satisfactory release. All structural curves required fixation and fusion. The mixture of autogenous and allogenic bone grafts was implanted for fusion.

A MAPM indicated that as many pedicle screws (or hooks) were placed as possible in the key vertebrae (api- cal vertebrae, end vertebrae, neutral vertebrae, and stable vertebrae) and adjacent to the key vertebrae to provide multiple anchor points (MAPs), which was based on the individual situation and actual intraoperative manipulation of each patient. However, due to severe dystrophic vertebral deformity and very thin pedicles (often existing in the area of structural curves), it was very difficult to place pedicle screws. To increase the number of screws and achieve MAPs, short pedicle screws that were shorter than normal (10-25 $\mathrm{mm}$ in length) were necessary to be placed depending on the intraoperative anatomical situation; normal pedicle screws could be cut to an appropriate length by a rod cutter or power cutter. In addition, shorter screws were not required to pass through the full length of the pedicle channel.

\section{Postoperative Procedure}

After correction, the neural system was examined. A Hemovac drain was usually removed when drainage flow was less than $10 \mathrm{ml}$ over 24 hours. The patients were mobilized earlier and exercises were begun after 12 days while wearing braces. Postoperatively, all patients received bracing therapy for an average of 6 months, and then were gradually weaned off the brace.

\section{Evaluation of Index and Statistical Analysis}

We obtained anteroposterior and lateral standing radiographs of the spine at the preoperative, postoperative, and final follow-up stages and measured the following parameters on these radiographs: coronal Cobb angle of the main curve, Cobb angle on the side-bending radiograph of the convex side, flexibility, correction rate, apical vertebral rotation (AVR), and sagittal kyphosis. The data were analyzed using SPSS (version 17.0, SPSS Inc.). Paired ttests were used to compare parameters at the preoperative, postoperative, and final follow-up stages. A p value $<0.05$ indicated a statistically significant difference.

\section{Results}

The mean follow-up period was 53 months (range 12-96 months). The mean postoperative coronal Cobb angle was $27.4^{\circ}$ (range $16.3^{\circ}-46.7^{\circ}$ ). Postoperatively, the mean AVR and angle of sagittal kyphosis were $1.2^{\circ}$ (range $1^{\circ}-2^{\circ}$ ) and $22.4^{\circ}$ (range $4.2^{\circ}-36.3^{\circ}$ ), respectively (Table 1 ). All patients showed good correction of all indices postoperatively, which revealed statistically significant differences between the postoperative and preoperative data ( $\mathrm{p}$ $<0.05$ for all). The mean postoperative correction rate was $58.7 \%$ (range $46.3 \%-74.1 \%$ ).

At the final follow-up evaluation, the mean coronal Cobb angle was $30.2^{\circ}$ (range $18.9^{\circ}-51.8^{\circ}$ ); the mean AVR and angle of sagittal kyphosis were $1.2^{\circ}\left(\right.$ range $1^{\circ}-2^{\circ}$ ) and $24.1^{\circ}$ (range $6.8^{\circ}-39.1^{\circ}$ ), respectively. All the indices also showed significant improvement at the final follow-up ( $\mathrm{p}$ $<0.05$ ). The mean follow-up correction rate was $56.4 \%$ (range 44.1\%-71.3\%), while the corrective loss rate of the Cobb angle was only $2.3 \%$ (Figs. 1 and 2; Table 1).

Shorter screws were placed in the vertebrae with severe dystrophic changes and very thin pedicles in all 31 patients. No severe complications such as spinal cord, neu- 
TABLE 1. Preoperative, postoperative, and final follow-up measurement data

\begin{tabular}{lccc}
\hline \multicolumn{1}{c}{ Measurement Data } & Preop & Postop & Final Follow-Up \\
\hline Coronal Cobb angle $\left(^{\circ}\right)$ & $69.1(48.9-91.4)$ & $27.4(16.3-46.7)^{*}$ & $30.2(18.9-51.8) \dagger$ \\
\hline Cobb angle $\left({ }^{\circ}\right)$ on the bending radiograph of the convex side & $58.2(40-79.8)$ & - & - \\
\hline Flexibility $(\%)$ & $15.6(8.3-28.2)$ & - & - \\
\hline Correction rate $(\%)$ & - & $58.7(46.3-74.1)$ & $56.4(44.1-71.3)$ \\
\hline AVR $\left(^{\circ}\right)$ & $2.5(2-3)$ & $1.2(1-2)^{*}$ & $1.2(1-2) \dagger$ \\
\hline Sagittal kyphosis & $58.3(34.1-79.6)$ & $22.4(4.2-36.3)^{*}$ & $24.1(6.8-39.1) \dagger$ \\
\hline
\end{tabular}

All values are given as mean (range). The postoperative and preoperative data as well as the final follow-up and preoperative data were analyzed using paired t-tests $(p<0.05=$ statistically significant difference) .

${ }^{*} p<0.05$ (postoperative vs preoperative).

$+p<0.05$ (final follow-up vs preoperative)

ral, or large vascular injury occurred during the operation. Furthermore, there were no cases of death or deep infection. In 2 patients, the incision for internal fixation was at a position higher than normal because the patients were thin, which caused skin compression and local pain. Only 1 patient presented with hook dislodgements because of poor fusion and pseudarthrosis, which required revision surgery.

\section{Discussion}

Regarding dystrophic scoliosis in patients with NF1, the main purpose of surgery is to stabilize the spine, prevent progression of deformity, and prevent nerve injury, rather than focus on the degree of correction. Some authors have already proved the need for early and aggressive surgical intervention for dystrophic scoliosis due to the inability of brace treatment to halt its progression. Furthermore, early spinal fusion does not lead to obvious loss of trunk height because the developing curves are usually short-segment curves, with limited growth potential.,12 Tsirikos et al. ${ }^{11}$ believe that dystrophic scoliotic curves associated with NF1 that are less than $20^{\circ}$ should be closely observed to identify any sudden rapid progression and thus prompt surgical management; for curves greater than $20^{\circ}$, posterior or anteroposterior instrumented fusion is strongly indicated. However, patients less than 10 years of age, when anteroposterior fusion is recommended, are in a peak growth period and posterior fusion alone may result in decompensation or the crankshaft phenomenon, because the anterior part of the spine keeps growing while the posterior part is fixed.

Treatment strategies remain controversial due to dystrophic vertebral deformity, very thin pedicles, osteoporosis, and rapid progression. ${ }^{7}$ Kim et al. ${ }^{6}$ believed that patients with a dystrophic scoliotic deformity of $20^{\circ}-40^{\circ}$ and kyphotic angle $<50^{\circ}$ were simply managed with posterior spinal instrumentation and fusion; to obtain better efficacy of correction and reduce the risk of pseudarthrosis, anteroposterior instrumented fusion was recommended as the most reliable surgical option in the presence of a dystrophic scoliotic deformity greater than $40^{\circ}$ or kyphotic angle greater than $50^{\circ}{ }^{11}$ However, other authors ${ }^{1,2}$ reported that the clinical efficacy of anteroposterior instrumented fusion was still not satisfactory due to severe hyperkyphosis and vertebral rotation in young patients. The potential presence of complex tumor or excessive plexiform venous channels around the vertebral bodies made the anterior approach impossible and a second attempt had to be made from the contralateral side, or even discontinued. Furthermore, because of the dystrophy, the apical vertebrae may have subluxated, or be so severely rotated that they are no longer in alignment with the rest of the spine. Such malalignment does not allow the anterior strut grafts to be placed in the concavity of the kyphosis and so they are mechanically ineffective in preventing its progression. ${ }^{4}$

It was found that the first-generation or second-generation internal fixation system was used in the majority of patients undergoing a posterior approach in the early years. A high incidence of internal fixation dislodgement, breakage, and pseudarthrosis was observed postoperatively. Moreover, due to obvious dystrophic changes and lack of good anchor points, it was very difficult to place posterior pedicle screws, which caused screws to be easily misplaced and fewer pedicle screws to be placed in the key vertebrae and adjacent to these key vertebrae. Parisini et al. ${ }^{10}$ reported that the failure incidence of posterior instrumented fusion alone and of the planned anterior and posterior fusion was 53\% and 23\%, respectively; regarding dystrophic spinal deformities accompanied by kyphosis, the failure incidence of the posterior instrumented fusion alone was $63 \%$. Other authors ${ }^{8}$ showed that the incidence of complications was $43.8 \%$, and that of pseudarthrosis was $6.3 \%$, following posterior instrumented fusion alone. However, in this study, very thin pedicles were revealed in all 31 patients suffering from dystrophic scoliosis associated with NF1. The mean preoperative coronal Cobb angle was $69.1^{\circ}$; the mean flexibility and AVR were $15.6 \%$ and $2.5^{\circ}$, respectively; and the mean angle of sagittal kyphosis was $58.3^{\circ}$. Posterior-only surgical correction was performed using the third-generation internal fixation system in all patients. During the operation, soft-tissue contractures and facet joint capsules were released widely and completely. As many pedicle screws (or hooks) were placed as possible to provide MAPs and spread out the corrective force. All patients showed good correction of all indices, and the mean postoperative correction rate was $58.7 \%$. At the final follow-up evaluation, the corrective loss rate of the Cobb angle was only $2.3 \%$. Only 1 patient required revision surgery because of pseudarthrosis and hook dislodgements. 

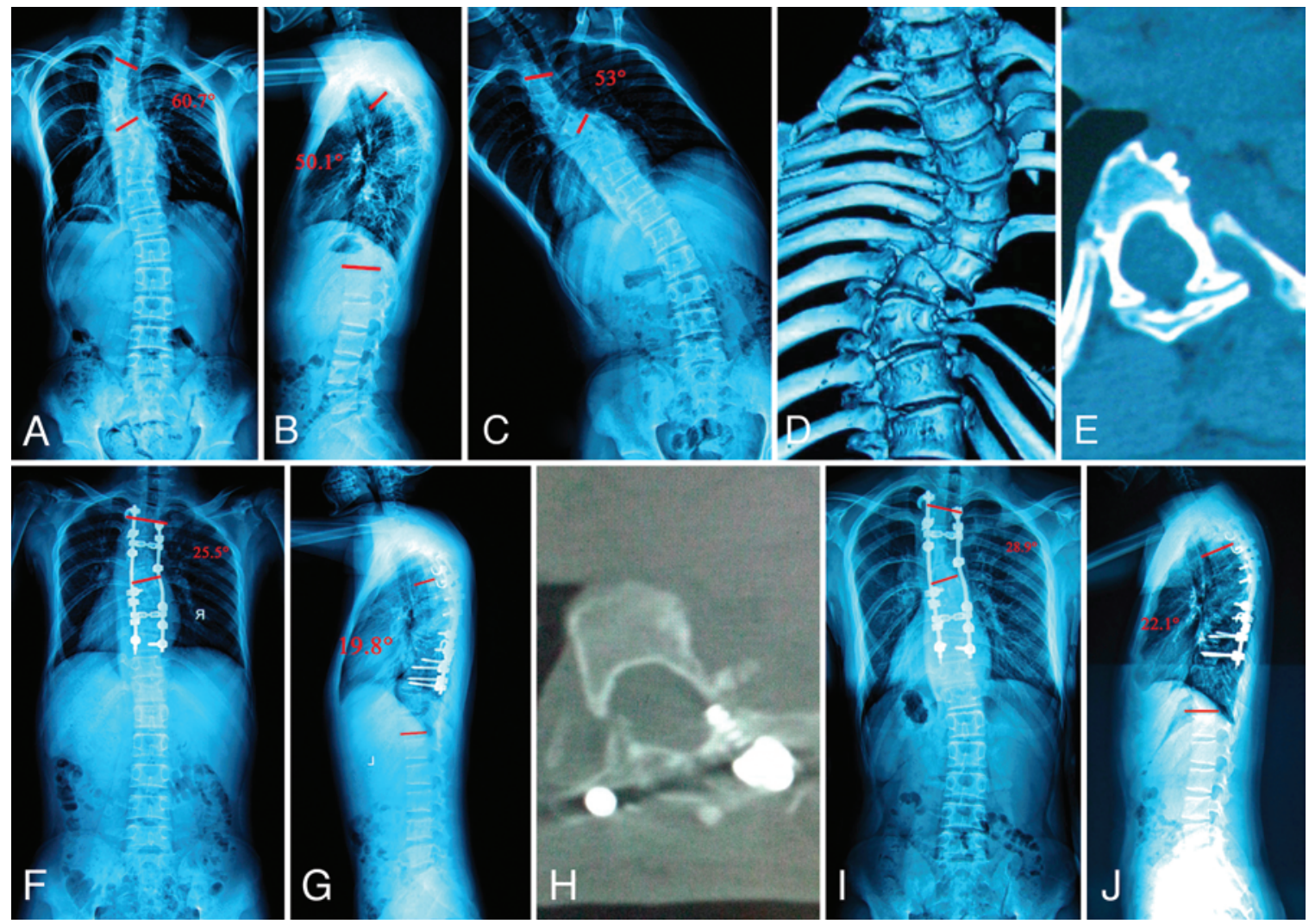

FIG. 1. Images obtained in a 14-year-old boy. A and B: Preoperative anteroposterior and lateral radiographs show that the coronal Cobb angle was $60.7^{\circ}$, the angle of sagittal thoracic kyphosis was $50.1^{\circ}$, and the AVR was $2^{\circ}$. C: Preoperative side-bending radiograph on the convex side shows that the Cobb angle was $53^{\circ}$ and flexibility was $12.8 \%$. D and E: Preoperative CT indicates obvious dystrophic changes, including vertebral scalloping, rib penciling, short angular curve, dural ectasia, and very elongated and thin pedicles. $\mathrm{F}$ and $\mathrm{G}$ : Postoperative anteroposterior and lateral radiographs show that the coronal Cobb angle was $25.5^{\circ}$, correction rate was $58 \%$, angle of sagittal thoracic kyphosis was $19.8^{\circ}$, and AVR was $1^{\circ}$. H: Postoperative CT indicates the MAPM using shorter posterior pedicle screws. I and J: Anteroposterior and lateral radiographs at 54 months after surgery show that the coronal Cobb angle was $28.9^{\circ}$, angle of sagittal thoracic kyphosis was $22.1^{\circ}$, and AVR was $1^{\circ}$. Figure is available in color online only.

Therefore, we deduced the following characteristics from our findings. First, due to poor bone quality and remarkable osteoporosis, the holding force of pedicle screws significantly decreased. Based on the individual situation and actual intraoperative manipulation of each patient, as many pedicle screws (or hooks) were placed as possible in the key vertebrae and adjacent to them to provide MAPs, spread out the corrective force, improve and stabilize the efficacy of correction, promote fusion, and reduce the incidence of pseudarthrosis.

Second, due to severe dystrophic vertebral deformity and very thin pedicles (often found in the area of structural curves), it was very difficult to place posterior pedicle screws, which caused screws to be easily misplaced. To increase the number of screws and achieve MAPs, short pedicle screws that were shorter than normal (10-25 mm in length) were necessary to be placed depending on the intraoperative anatomical situation; normal pedicle screws could be cut to an appropriate length by a rod cutter or power cutter. In addition, shorter screws were not required to pass through the full length of the pedicle channel, which could reduce the incidence of complications such as misplaced screws, leakage of CSF, and neurovascular injury.
Third, in this study, rigid scoliosis was observed in all patients, and the mean flexibility was only $15.6 \%$. During the operation, soft-tissue contractures and facet joint capsules were necessary to be released widely and completely. If necessary, parts of the ribs and transverse processes at the apical vertebrae were removed for increasing spinal flexibility.

Fourth, an adequate mixture of autogenous and allogenic bone grafts should be implanted for fusion, and all facet joints need to be carefully fused to reduce the risk of pseudarthrosis. And fifth, because of a thin vertebral lamina in dystrophic scoliosis, careful exposure is required during the operation, and when decorticating for fusion, accidentally sliding the osteotome into the spinal canal needs to be prevented.

\section{Conclusions}

The level of difficulty for this operation was higher, and the strategies were more complicated, for the treatment of dystrophic scoliosis associated with NF1 than that of idiopathic scoliosis. However, posterior-only surgical correction of dystrophic scoliosis in patients with NF1 using a MAPM could yield satisfactory clinical efficacy of correc- 

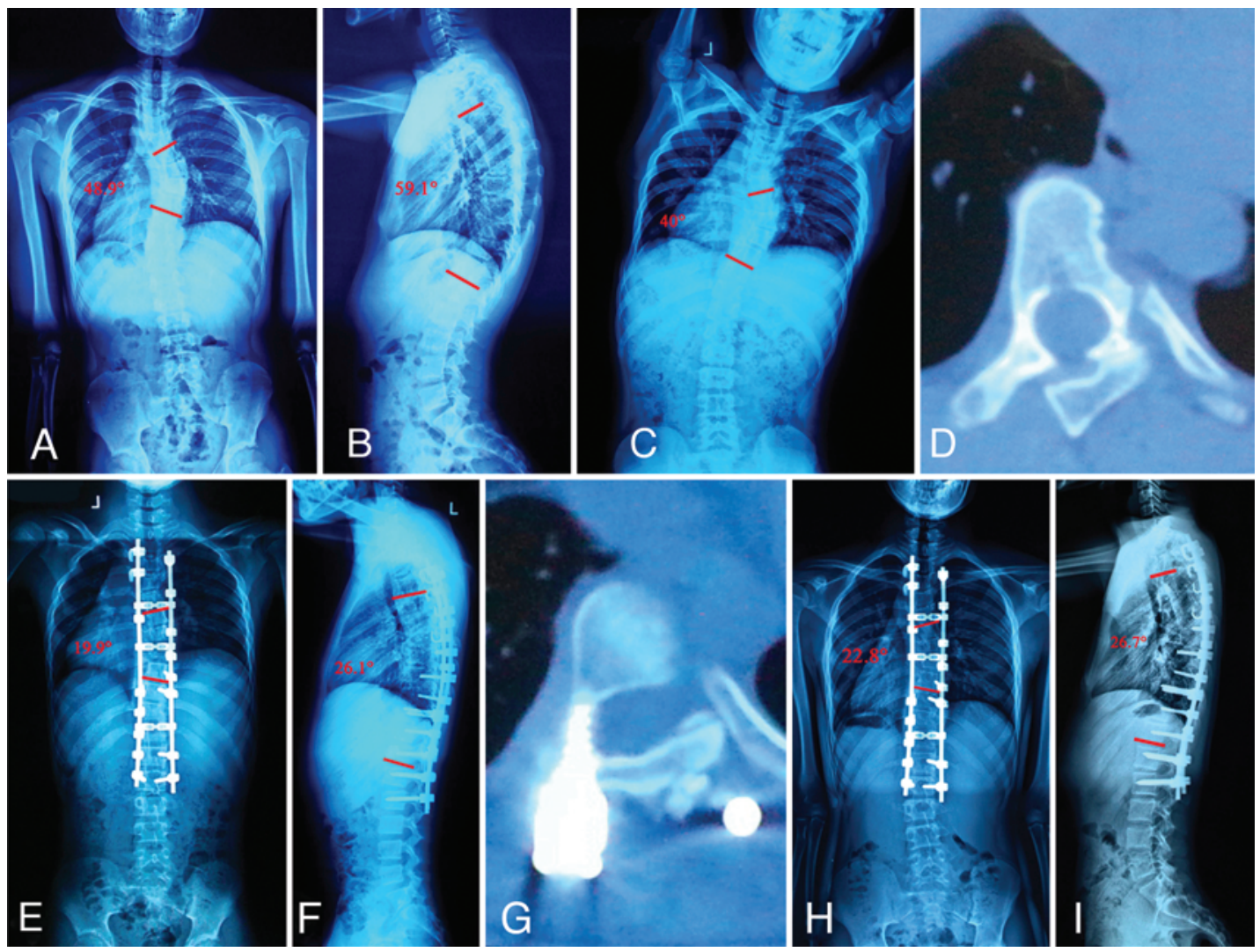

FIG. 2. Images obtained in a 13-year-old boy. A and B: Preoperative anteroposterior and lateral radiographs show that the coronal Cobb angle was $48.9^{\circ}$, angle of sagittal thoracic kyphosis was $59.1^{\circ}$, and AVR was $2^{\circ}$. C: Preoperative side-bending radiograph on the convex side shows that the Cobb angle was $40^{\circ}$ and flexibility was $18.2 \%$. D: Preoperative CT indicates obvious dystrophic changes including vertebral scalloping, dural ectasia, and very elongated and thin pedicles. E and F: Postoperative anteroposterior and lateral radiographs show that the coronal Cobb angle was $19.9^{\circ}$, correction rate was $59.3 \%$, angle of sagittal thoracic kyphosis was $26.1^{\circ}$, and AVR was $1^{\circ}$. G: Postoperative CT indicates the MAPM using shorter posterior pedicle screws. $\mathrm{H}$ and I: Anteroposterior and lateral radiographs at 36 months after surgery show that the coronal Cobb angle was $22.8^{\circ}$, angle of sagittal thoracic kyphosis was $26.7^{\circ}$, and AVR was $1^{\circ}$. Figure is available in color online only.

tion and fusion in reducing the incidence of complications. Furthermore, the primary goal of surgery in those cases is to stabilize the vertebral column and halt further progression of the deformity rather than perform heroic attempts for correction that could potentially result in permanent neurological injuries. Appropriate surgical candidates should be carefully selected prior to any procedure; inclusive preoperative evaluation and sufficient intraoperative exposure and monitoring are necessary to improve surgical outcomes.

\section{Acknowledgments}

The study was supported by the Natural Science Foundation of Hunan Province, China (grant no. 2016JJ3160), and the National Natural Science Foundation of China (grant no. 81472145).

\section{References}

1. Crawford AH: Pitfalls of spinal deformities associated with neurofibromatosis in children. Clin Orthop Relat Res (245):29-42, 1989

2. Crawford AH, Herrera-Soto J: Scoliosis associated with neurofibromatosis. Orthop Clin North Am 38:553-562, vii, 2007
3. Feldman DS, Jordan C, Fonseca L: Orthopaedic manifestations of neurofibromatosis type 1. J Am Acad Orthop Surg 18:346-357, 2010

4. Hsu LC, Lee PC, Leong JC: Dystrophic spinal deformities in neurofibromatosis. Treatment by anterior and posterior fusion. J Bone Joint Surg Br 66:495-499, 1984

5. Jaremko JL, MacMahon PJ, Torriani M, Merker VL, Mautner VF, Plotkin SR, et al: Whole-body MRI in neurofibromatosis: incidental findings and prevalence of scoliosis. Skeletal Radiol 41:917-923, 2012

6. Kim HW, Weinstein SL: Spine update. The management of scoliosis in neurofibromatosis. Spine (Phila Pa 1976) 22:2770-2776, 1997

7. Koptan W, ElMiligui Y: Surgical correction of severe dystrophic neurofibromatosis scoliosis: an experience of 32 cases. Eur Spine J 19:1569-1575, 2010

8. Li M, Fang X, Li Y, Ni J, Gu S, Zhu X: Successful use of posterior instrumented spinal fusion alone for scoliosis in 19 patients with neurofibromatosis type- 1 followed up for at least 25 months. Arch Orthop Trauma Surg 129:915-921, 2009

9. Lykissas MG, Schorry EK, Crawford AH, Gaines S, Rieley M, Jain VV: Does the presence of dystrophic features in patients with type 1 neurofibromatosis and spinal deformities increase the risk of surgery? Spine (Phila Pa 1976) 38:1595-1601, 2013

10. Parisini P, Di Silvestre M, Greggi T, Paderni S, Cervellati S, 
Savini R: Surgical correction of dystrophic spinal curves in neurofibromatosis. A review of 56 patients. Spine (Phila Pa 1976) 24:2247-2253, 1999

11. Tsirikos AI, Saifuddin A, Noordeen MH: Spinal deformity in neurofibromatosis type-1: diagnosis and treatment. Eur Spine J 14:427-439, 2005

12. Wang Z, Liu Y: Research update and recent developments in the management of scoliosis in neurofibromatosis type 1. Orthopedics 33:335-341, 2010

\section{Disclosures}

The authors report no conflict of interest concerning the materials or methods used in this study or the findings specified in this paper.

\section{Author Contributions}

Conception and design: Zhang, Deng. Acquisition of data: Deng, Tang, Liu, Wang. Analysis and interpretation of data: Deng,

Tang, Liu, Wang. Drafting the article: Deng. Critically revising the article: Zhang, Deng, Gao. Reviewed submitted version of manuscript: Zhang, Deng, Gao. Approved the final version of the manuscript on behalf of all authors: Zhang. Statistical analysis:

Tang, Liu, Wang, Gao. Administrative/technical/material support: Tang, Liu, Wang, Gao. Study supervision: Zhang, Gao.

\section{Correspondence}

Hong-Qi Zhang, Department of Spine Surgery, Xiangya Hospital of Central South University, 87 Xiangya Rd., ChangSha 410008, China. email: zhanghq9996@126.com. 